\title{
On Robust Multi-Year Tidal Prediction Using T_TIDE
}

\author{
Do-Seong Byun ${ }^{1 *}$ and Deirdre Erin Hart ${ }^{2}$ \\ ${ }^{1}$ Ocean Research Division, Korea Hydrographic and Oceanographic Agency, Busan 49111, Korea \\ ${ }^{2}$ Department of Geography, College of Science, University of Canterbury, Christchurch 8041, New Zealand
}

Received 8 July 2019; Revised 4 September 2019; Accepted 9 September 2019

(c) KSO, KIOST and Springer 2019

\begin{abstract}
$\overline{\text { Abstract - A minimum } 19 \text { year tidal prediction dataset covering }}$ nodal (satellite) modulation effects is required to determine the Lowest Astronomical Tide (LAT) and Highest Astronomical Tide (HAT) datums. In this study, we explore the ability of a widely used conventional standard harmonic prediction program, T_TIDE 't predic.m' from Pawlowicz et al. (2002), to produce accurate continuous multi-year predictions. Comparisons are made with the more recent tidal prediction program, UTide 'ut reconstr.m' from Codiga (2011). Tidal height records for two different regimes are employed: for diurnal tides data are employed from Cape Roberts in Antarctica, while for semi-diurnal tides data are used from Incheon, Gyeonggi Bay, Korea. Results demonstrate an issue arises in continuous multi-year tidal predictions made via T_TIDE, due to the program's single calculation (fixed) of nodal modulation corrections (NMC). We explain a modified NMC update method that successfully solves this problem, rendering the program of use for accurate continuous multi-year tidal predictions.
\end{abstract}

Keywords - tidal harmonic prediction, nodal factors and nodal angles, update period of nodal modulation corrections

\section{Introduction}

Many human activities in coastal environments rely on sea level information derived from long-term tidal predictions, including safe ship navigation and coastal planning. Such predictions are of use in determining nautical chart elevations (i.e. reference depth or height datums) and hydrographic forecasts (i.e. tide levels). A recent resolution of the IHO (2018) specified the adoption of Lowest Astronomical Tide (LAT) and Highest Astronomical Tide (HAT), or very similar levels, for official chart datums and vertical clearances. LAT and HAT datums are used in many countries, including in Australia,

\footnotetext{
*Corresponding author. E-mail: dsbyun@korea.kr
}

France, New Zealand, the United Kingdom and the United States of America. The IHO (2018) further recommended that "LAT and HAT should be calculated over a minimum period of 19 years using harmonic constants derived from a minimum of one year's observations or by other proven methods known to give reliable results". If 19 years of continuous, high-quality observations are available for any particular site, it is of course preferable to derive datums from those data, thereby avoiding predicted tidal potential values and instead directly deriving the 'satellite constituents' whose amplitudes and phases underlie the astronomical arguments, nodal factors and nodal angles. From a practical standpoint, however, the use of 19 year tidal predictions to determine LAT and HAT is often logical, since it is difficult to obtain 19 seamless years of high quality sea level observation records. More often than not, records are interrupted by missing data windows, data spikes, observation station re-locations, and other issues.

The harmonic analysis and prediction MATLAB package 'T_TIDE' was developed by Pawlowicz et al. (2002) as a user-friendly MATLAB version of the theoretical ideas represented in Foreman's (1977) Fortran-based, Institute of Ocean Sciences (IOS) tidal package. T_TIDE contains separate programs for tidal prediction ('t predic.m') and harmonic analysis ('t_tide.m'). Subsequently, Codiga (2011) was built on the foundations of T_TIDE, integrating concepts from Leffler and Jay (2009) and Foreman et al. (2009), to produce the 'Unified Tidal analysis and prediction' package or UTide. Despite Codiga's (2011) significant advance, T_TIDE remains the most widely used tidal analysis and prediction package today: as a rough indication of research uptake, a 2019 Google Scholar search for Pawlowicz et al. (2002) returned $>2000$ citations whereas one for Codiga (2011) returned $>100$ 
citations. Note that the astronomical arguments, nodal factors and nodal angles used in T_TIDE, UTide and IOS software are based on tidal potential values (e.g., Cartwright and Tayler 1971; Cartwright and Edden 1973).

With respect to long-term tidal harmonic analysis, Pawlowicz (2011) recommended use of single-year data slices when analyzing time series between 1 and 18.6 years in length in 't tide.m'. Codiga (2011) pointed out that conventional tidal harmonic analysis methods, such as 't_tide.m', were not suited to single-run analyses of multi-year records due to their use of fixed nodal (satellite) Modulation Corrections (NMC). UTide was developed to overcome this and several other issues (Codiga 2011).

Unlike for 't tide.m', which comes with a website warning regarding multi-year analyses, no parallel constraints are specified for the use of T_TIDE's 't predic.m'. There has been little examination of the accuracy of this prediction program in producing multi-year data sets. Given the continued ubiquity of T_TIDE use, and the above-mentioned IHO (2018) recommendation, this study aims to examine the usefulness of T_TIDE for generating 19-year prediction data sets in order to analyze LAT and HAT elevations. Here we show that, unlike the UTide 'ut_reconstr.m' program, the T_TIDE 't predic.m' program should not be used for producing multi-year (e.g. 19 year) continuous tidal predictions in its current form, due to a similar NMC issue as occurs in 't tide.m'. Fortunately, we have been able to develop a simple program modification for ' $t$ _ predic.m', enabling its use for producing accurate 19 year predictions.

\section{Background on Classical Tidal Harmonic Prediction Parameterization}

Tidal heights $h$ at any time $\tau$ can be predicted with relative accuracy for a given station from the superposition of the sinusoidal tidal harmonic constituent amplitudes $\left(a_{i}\right)$ and phase-lags $\left(g_{i}\right)$, together with their astronomical arguments $\left(V_{i}\right)$, nodal factors $\left(f_{i}\right)$ and nodal angles $\left(u_{i}\right)$ derived from harmonic analysis of year-long sea-level records, as expressed by:

$$
h(\tau)=\sum_{i=1}^{n} f_{i}(\tau) a_{i} \cos \left[\omega_{i} t+V_{i}\left(t_{0}\right)+u_{i}(\tau)-g_{i}\right]
$$

where $\tau$ is the reference time $\left(t_{0}\right)$ plus the time $(t)$ elapsed since $t_{0}$ (i.e., $\tau=t_{0}+t$ ); $n$ is the number of constituents; the subscript $i$ denotes each tidal constituent; and $\omega_{i}$ indicates the angular speeds $\left({ }^{\circ} \mathrm{hr}^{-1}\right)$ of the tidal constituents.

While in reality $f_{i}$ and $u_{i}$ vary slowly over the entire 18.61 year nodal cycle, their calculation for any given period of time can be computationally onerous. To improve calculation efficiency, classical tidal prediction programs simply update the values used to represent these factors and angles at specified intervals. For example, the 'marie.f' program of the Task2000 package by Bell et al. (1999???) uses constant values for $f_{i}$ and $u_{i}$ that are re-calculated every three days in order to predict tidal heights according to:

$$
h(\tau)=\sum_{t=1}^{n} f_{i}\left(\tau_{3}\right) a_{i} \cos \left[\omega_{i} t+V_{i}\left(t_{0}\right)+u_{i}\left(\tau_{3}\right)-g_{i}\right]
$$

Similarly, for prediction records $>1$ month, the IOS 'tide 4.f' program uses values of $f_{i}$ and $u_{i}$ computed on the 16th day of each prediction month to produce tidal height predictions, as given by:

$$
h(\tau)=\sum_{i=1}^{n} f_{i}\left(\tau_{16}\right) a_{i} \cos \left[\omega_{i}\left(\tau-\tau_{16}\right)+V_{i}\left(\tau_{16}\right)+u_{i}\left(\tau_{16}\right)-g_{i}\right]
$$

Unlike in its IOS predecessor, values of $f_{i}$ and $u_{i}$ are calculated only once in T_TIDE's 't predic.m' (regardless of prediction length), in the middle $\left(\tau_{\text {mid }}\right)$ of the prediction period, as expressed by:

$$
h(\tau)=\sum_{i=1}^{n} f_{i}\left(\tau_{\text {mid }}\right) a_{i} \cos \left[\omega_{i}\left(\tau-\tau_{\text {mid }}\right)+V_{i}\left(\tau_{\text {mid }}\right)+u_{i}\left(\tau_{\text {mid }}\right)-g_{i}\right]
$$

Note that in 't _predic.m' Eq. (4) is expressed in a complex form, so that it is capable of using both scalar (sea-level) and vector (currents) data (Pawlowicz et al. 2002).

\section{Experimental Settings}

19 year tidal experiments comparing the prediction capabilities of T_TIDE (Pawlowicz et al. 2002) and UTide (Codiga 2011) were conducted using data from two tidal observation stations with different tidal characteristics: Cape Roberts (ROBT), located in Terra Nova Bay on Antarctica's Ross Sea coast, with its diurnal, micro-tidal regime (Form factor $F=4.1$ ); and Incheon, located in Gyeonggi Bay on Korea's Yellow Sea coast, with its semi-diurnal, macro-tidal regime (Form factor $F=0.17$ ) (Fig. 1; Table 1).

There are three key ways to derive the tidal harmonic constants required for calculating LAT and HAT over a 19 year period: these are from 1) harmonic analysis of one year 
(a) Cape Roberts (ROBT)
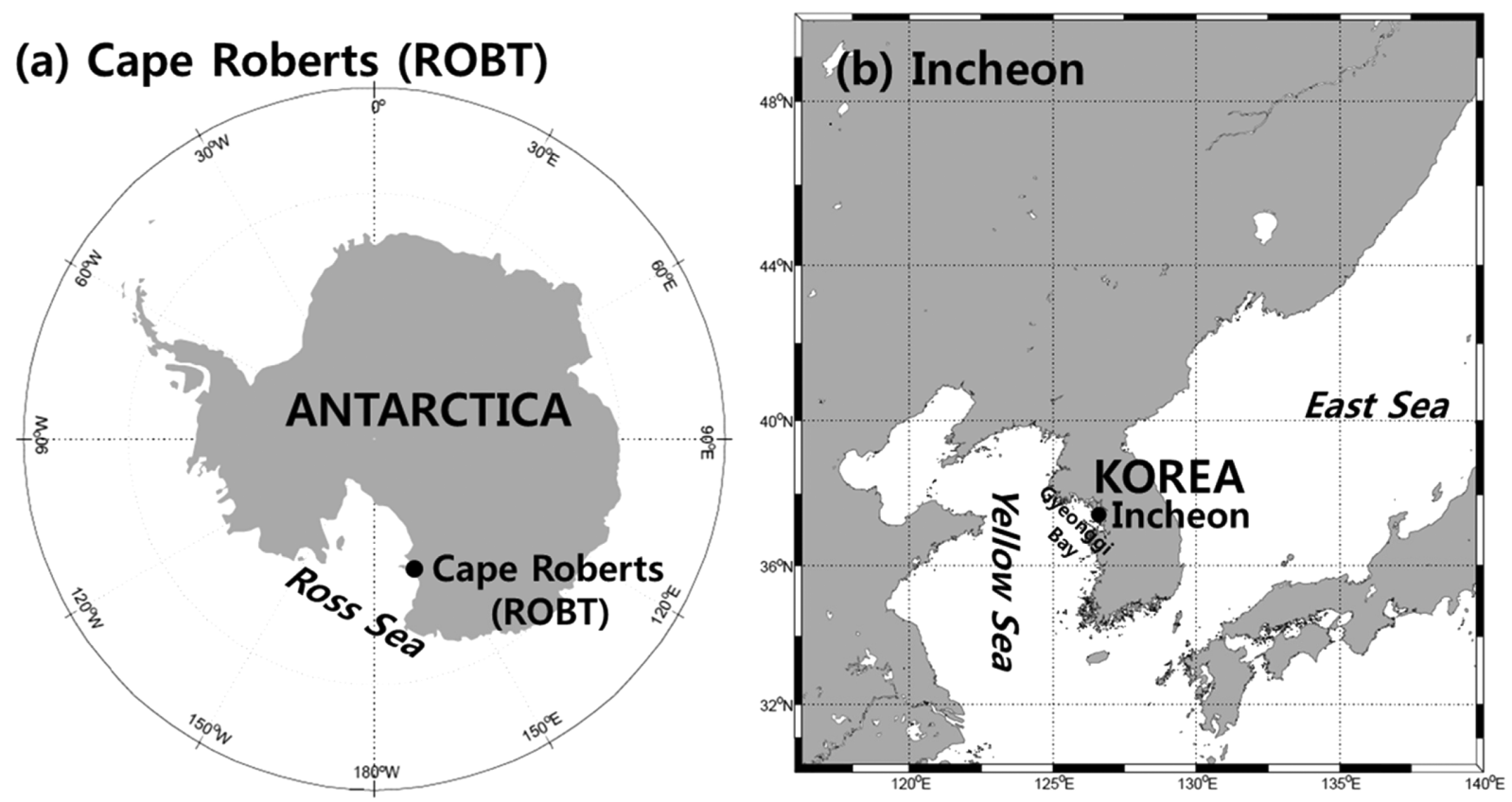

Fig. 1. Maps showing locations of the tidal observation stations ( and of Incheon in Gyeonggi Bay, Korea

) of Cape Roberts (ROBT) in Tera Nova Bay, Ross Sea, Antarctica

Table 1. Tidal harmonic analysis results for four major constituents, derived from yearlong observations recorded at Cape Roberts, Antarctica (2013) and Incheon, Korea (2016). Phase-lags are referenced to Greenwich Mean Time $(G)$ for Cape Roberts, and to the $135^{\circ} \mathrm{E}$ time zone $(g)$ for Incheon, respectively. $F$ indicates the tidal form factor (Courtier 1938)

\begin{tabular}{|c|c|c|c|c|c|c|c|c|c|}
\hline \multirow{3}{*}{ Station } & \multicolumn{4}{|c|}{ Diurnal tides } & \multicolumn{4}{|c|}{ Semi-diurnal tides } & \multirow{3}{*}{$F=$} \\
\hline & \multicolumn{2}{|c|}{$\mathrm{K}_{1}$} & \multicolumn{2}{|c|}{$\mathrm{O}_{1}$} & \multicolumn{2}{|c|}{$\mathrm{M}_{2}$} & \multicolumn{2}{|c|}{$\mathrm{S}_{2}$} & \\
\hline & $a_{K_{1}}(\mathrm{~cm})$ & $G_{K_{1}}\left({ }^{\circ}\right)$ & $a_{O_{1}}(\mathrm{~cm})$ & $G_{O_{1}}\left({ }^{\circ}\right)$ & $a_{M_{2}}(\mathrm{~cm})$ & $g_{M_{2}}\left({ }^{\circ}\right)$ & $a_{S_{2}}(\mathrm{~cm})$ & $g_{S_{2}}\left({ }^{\circ}\right)$ & \\
\hline Cape Roberts (ROBT) & 20.5 & 217 & 21.1 & 202 & 5.3 & 5 & 4.9 & 309 & $F=4.1$ (Diurnal and microtidal) \\
\hline Incheon & 39.4 & 303 & 29.0 & 264 & 282.8 & 130 & 111.8 & 187 & $F=0.17$ (Semi-diurnal and macrotidal) \\
\hline
\end{tabular}

observation records; 2) the vector average of tidal harmonic constants produced via analysis of nineteen sequential yearlong slices of observation records; and 3) harmonic analysis of 19 year observation records. We used the first of these approaches to obtain the tidal harmonic constants needed for our prediction experiments. Year-long, 5 and 10 minute interval sea level records were obtained for the Cape Roberts (ROBT) (2013????) and Incheon (2016???) tidal stations, from Land Information New Zealand (LINZ) and the Korea Hydrographic and Oceanographic Agency (KHOA), respectively. These records were harmonically analyzed using both T_TIDE's 't tide.m' and UTide's 'ut_solv.m' programs (see Table 1 for selected harmonic analysis results). Then in simplified experiments, we employed just the four main tidal harmonic constants $\mathrm{M}_{2}, \mathrm{~S}_{2}, \mathrm{~K}_{1}, \mathrm{O}_{1}$ in the two different prediction programs, T_TIDE's 't predic.m' and UTide's 'ut_reconstr.m', to generate hourly tidal heights for the 19 year period from 2015 to 2033 . Note that tidal heights are typically predicted at 1 minute intervals in order to calculate LAT and HAT. Excluding the contributions of the longer-term $\mathrm{S}_{a}$ and $\mathrm{S}_{s a}$ constituents, the four constituents' tidal amplitudes employed in the simplified experiments account for $55 \%$ and $66 \%$ of the total tidal amplitudes at Cape Roberts and Incheon, respectively.

\section{Results and Discussion}

\section{Accuracy of T_TIDE versus UTide generated 19 year tidal predictions}

In the 19 year T_TIDE generated continuous predictions, hourly tidal height variation remained constant for both stations, with no evidence of the 18.61 year nodal cycle (Fig. $2 \mathrm{a}, \mathrm{b})$. In contrast, the UTide predictions clearly exhibited inter-annual variation due to the 18.61 year nodal cycle (Fig. 

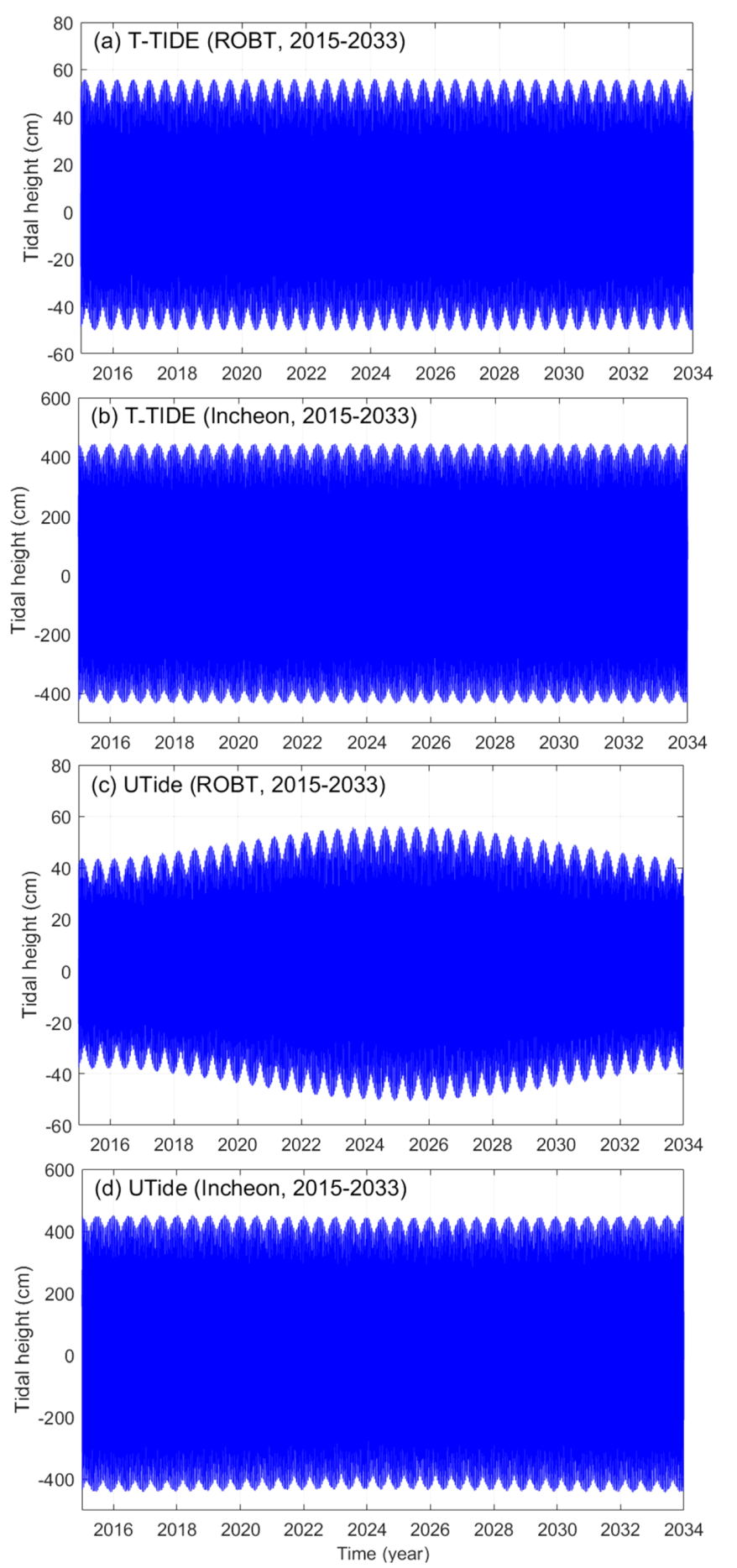

Fig. 2. Comparison of 19 year, continuous tidal height time series predicted for Cape Roberts (ROBT: a, c) and Incheon tidal stations $(b, d)$, using T_TIDE's 't predic.m $(a, b)$ versus UTide's 'ut reconstr.m' (c, d), with the tidal harmonic constants of the four major constituents $\left(\mathrm{K}_{1}, \mathrm{O}_{1}, \mathrm{M}_{2}, \mathrm{~S}_{2}\right)$ as input data

2c, d). These results indicate that T_TIDE should not be used, in its current form, to generate continuous multi-year tidal predictions. Fig. 2c and d also reveals that the long-term nodal cycle variation in tidal heights is more pronounced in diurnal regimes, like that of Cape Roberts, than in semidiurnal regimes, such as at Incheon.

The question arises as to which part of T_TIDE's current prediction parameterization hinders the accurate generation of long-term tidal height variation. Other questions arising are: why does the diurnal regime of Cape Roberts exhibit greater inter-annual tidal variation than the semi-diurnal regime of Incheon, and why are there tidal envelope variability differences between diurnal and semi-diurnal tidal regimes across the 18.61 year nodal cycle?

Firstly, in tidally-dominated coastal environments according to Byun and Cho (2009), the accurate generation of 18.61 year tidal variations depends on the NMC update period for the lunar constituents. Thus, we paid close attention to the part of the T_TIDE 't _ predic.m' code that deals with NMC. Specifically, the 't_vuf.m' function is employed to calculate the astronomical argument $(V)$ and nodal factors and angles $\left(f_{i}, u_{i}\right)$ for each tidal constituent. Regardless of the length of the entire tidal prediction period (ntim), 't_vuf.m' is called just once in 't predic.m', at the midpoint of the prediction period $\left(j d_{\text {mid }}\right)$ (Fig. 3a). [Note that this is similar to in 't tide.m', where the NMC are calculated for the middle day of the entire data record being harmonically analyzed.] In 't predic.m', the fixed $\operatorname{NMC}\left(f_{i}, u_{i}\right)$ for each tidal constituent are then utilized for the whole prediction period, leading to inaccurate continuous multi-year tidal predictions.

Additional 't predic.m' experiments were performed to generate individual year-long hourly tidal predictions from 2015 to 2033, using the four major tidal harmonic constants $\left(\mathrm{K}_{1}, \mathrm{O}_{1}, \mathrm{M}_{2}, \mathrm{~S}_{2}\right)$, with results concatenated to produce one nineteen year prediction series. The resulting tidal height time series exhibited inter-annual variation across the 18.61 year nodal cycle, plus a consistent pattern of within-year tidal height variation for each diurnal and semidiurnal tide at ROBT and Incheon (Fig. 4a, d). Breaking the results down further, for both sites the diurnal (Fig. 4b, e) and semi-diurnal (Fig. 4c, f) components of the predicted tides exhibited yearly stepped variations across the prediction period. As shown in Fig. 5a and $b$, these annual steps are an artefact of the 't predic.m' program's once-per-year calculation of the NMC for the three major lunar constituents, in the experimental set-up. To illustrate this issue further, we estimated daily NMC values over the nineteen year prediction period for the ROBT site from UTide's 'ut_reconstr.m'. Note that 'ut_FUV' used in 
(a) Original calculation procedure of $(\mathrm{V}, \mathrm{u}, \mathrm{f})$ in $\mathrm{t}_{-} \mathrm{vuf} . \mathrm{m}$ for tidal prediction

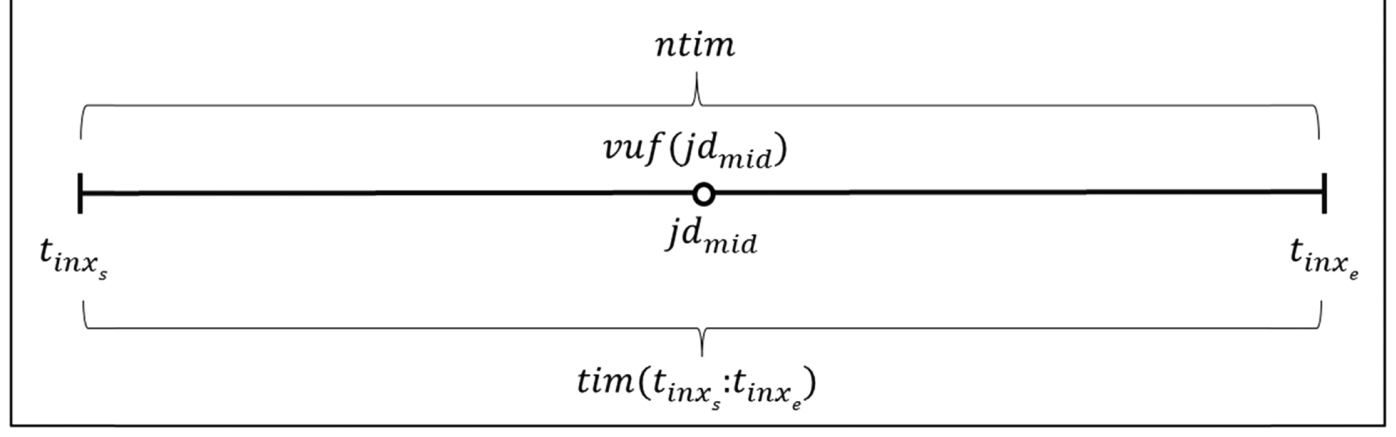

\section{(b) Modified calculation procedure of $(\mathrm{V}, \mathrm{u}, \mathrm{v})$ in $\mathrm{t}_{-} \mathrm{vuf} . \mathrm{m}$ for tidal prediction}

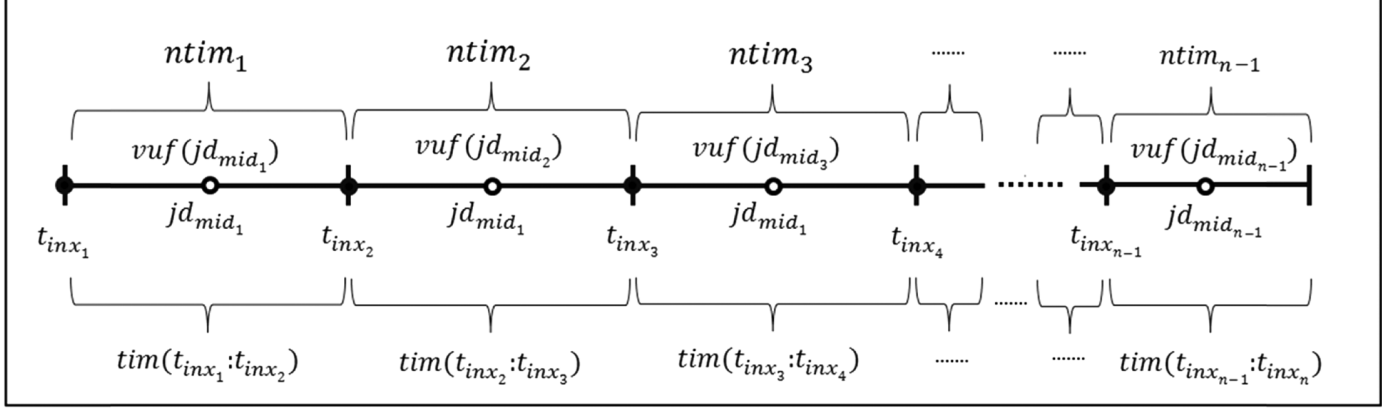

Fig. 3. Schematic showing the (a) original and (b) modified nodal modulation correction (NMC) calculation procedures in $t$-vuf.m for tidal prediction. 'tim' and 'ntim' are the time and the length of ' $t_{\text {inx }}$ ', the between-time index; ' $j d_{\text {mid }}$ ' is the middle day of 'tim'; and $v u f\left(j d_{\text {mid }}\right)$ represents the values of the $\mathrm{NMC}$ at $j d_{\text {mid }}$

'ut_reconstr.m' is based on T_TIDE's 't_vuf.m' (Codiga 2011), with the nodal factors and nodal angles being largely unaffected by latitude. In contrast to the T_TIDE experiments, results from UTide's 'ut_reconstr.m' exhibited smooth and continuous variation in nodal factors and nodal angles over the entire prediction period (Fig. $5 \mathrm{c}, \mathrm{d}$ ), indicating that UTide is perfectly able to generate continuous multi-year tidal predictions. Together these experimental results confirm our hypothesis that the 't predic.m' program's fixed NMC update period is the problematic element of T_TIDE that hinders accurate multi-year predictions, as earlier illustrated in Fig. 2a and $b$. Note that despite T_TIDE being based on the theory contained in Foreman's (1977) IOS tidal package, the NMC update period in T_TIDE's 't predic.m' does not match that of the IOS package, which updates the NMC using values on the sixteenth day of each prediction month.

Next, we explored the reason why there was a greater degree of inter-annual tidal variation exhibited in the Cape Roberts diurnal regime than in the Incheon semi-diurnal regime, with out-of-phase inter-annual tidal variations between the diurnal $\left(\mathrm{K}_{1}\right.$ and $\left.\mathrm{O}_{1}\right)$ versus semi-diurnal $\mathrm{M}_{2}$ constituents across the 18.61 year cycle. Fig. $5 \mathrm{c}-\mathrm{d}$ compares cyclic variation in the NMC factors $\left(f_{i}\right.$ and $u_{i}$ ) of three key constituents for Cape Roberts. The cyclic variations in nodal factors for the diurnal $\mathrm{O}_{1}$ and $\mathrm{K}_{1}$ tidal constituents were markedly greater than those of the semi-diurnal $\mathrm{M}_{2}$ tide. The total nodal factor value range (and the minimum to maximum correction factors) was 0.3839 (and 0.8006 to 1.1839 ) for the $\mathrm{O}_{1} ; 0.2320$ (and 0.8813 to 1.1132 ) for the $\mathrm{K}_{1}$; and 0.0754 (and 0.9628 to 1.0382) for the $\mathrm{M}_{2}$, respectively (Fig. 5c). These results indicate that the maximum tidal ranges experienced at the Cape Roberts site will vary considerably as a result of interannual changes in the diurnal nodal correction factors over an 18.61 year cycle. In contrast, the predominantly semi-diurnal tides of Incheon Station experience proportionally smaller inter-annual changes in tidal range, since the $\mathrm{M}_{2}$ nodal factor varies little (about $\pm 4 \%$ ) over 18.61 years. Yet, given the macro-tidal nature of this site, even proportionally small 

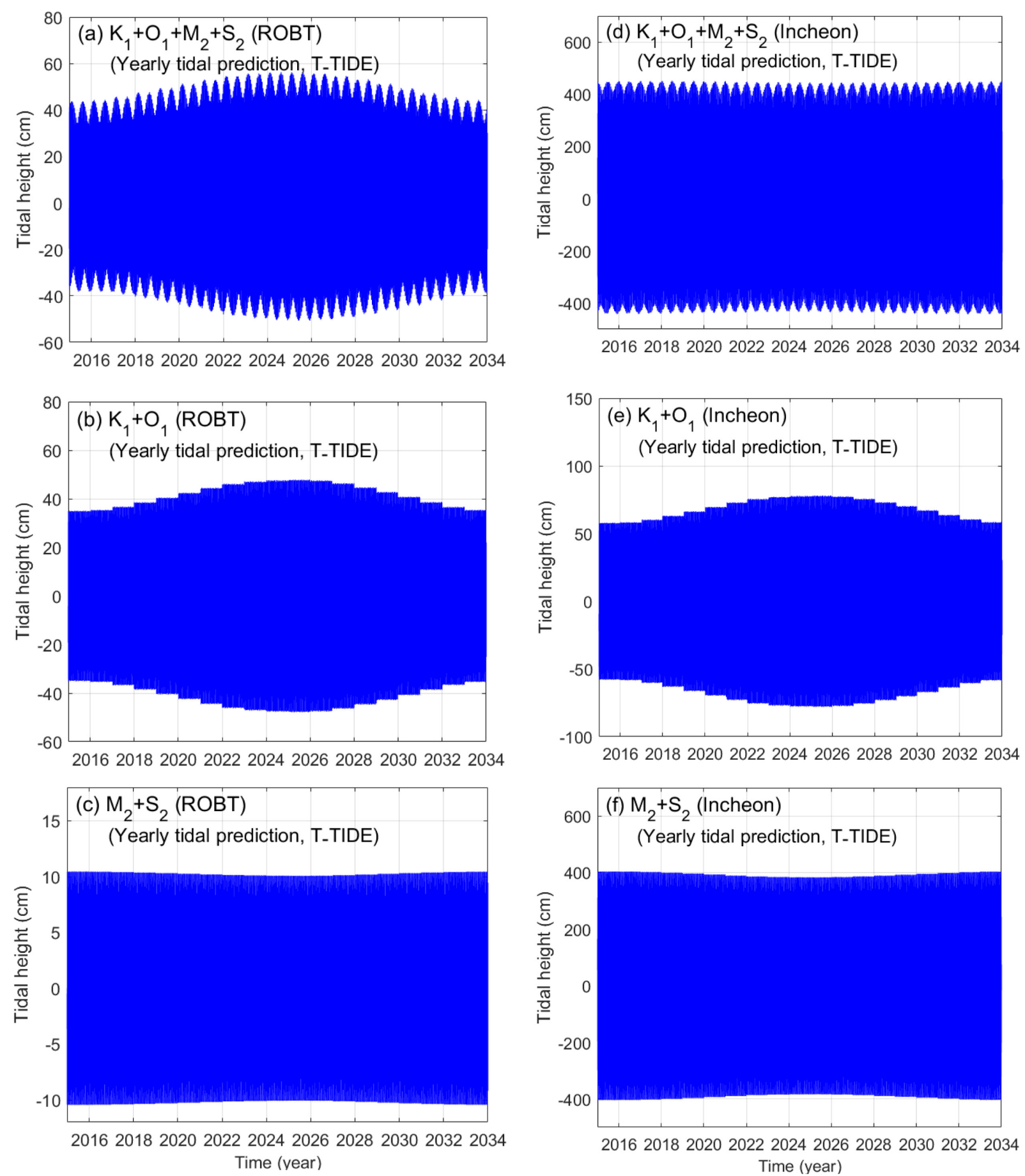

Fig. 4. 19 year concatenated time series of annual, hourly-interval tidal height predictions using T_TIDE's ' $t$ ppredic.m' and the four major tidal harmonic constants (a,d); the $\mathrm{K}_{1}$ and $\mathrm{O}_{1}$ harmonic constants only $(\mathrm{b}, \mathrm{e})$; and the $\overline{\mathrm{M}}_{2}$ and $\mathrm{S}_{2}$ harmonic constants only $(\mathrm{c}, \mathrm{f})$ at $\operatorname{ROBT}(\mathrm{a}, \mathrm{b}, \mathrm{c})$ and Incheon $(\mathrm{d}, \mathrm{e}, \mathrm{f})$ tidal stations, respectively

NMC variations can cause sea-level differences of $>30 \mathrm{~cm}$, meaning that such effects should not be ignored.

Additional experiments were conducted, similar to those illustrated in Fig. 2a and b), where the individual lunar tidal constituents' $\operatorname{NMC}\left(f_{i}\right.$ and $\left.u_{i}\right)$ were calculated for the middle day of the nineteen year prediction period (June 2, 2024), and used one-by-one in T_TIDE's 't predic.m' to generate 2015 to 2033 tidal height predictions. In these results, the diurnal and the semi-diurnal tides were overestimated and underestimated, respectively. These inaccuracies occurred 

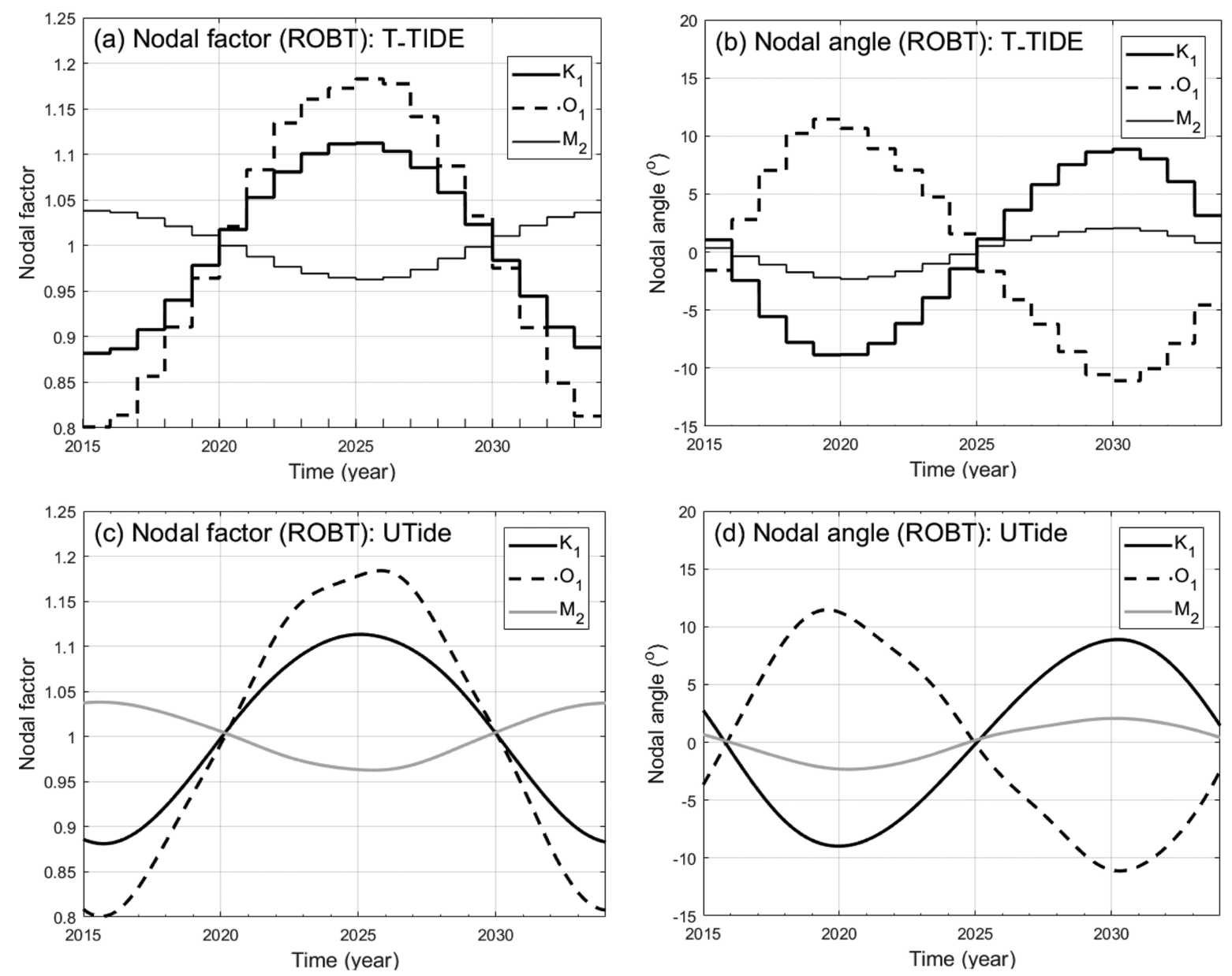

Fig. 5. Variation in the nodal factors ( $a, c)$ and nodal angles $(b, d)$ of the $K_{1}, O_{1}$ and $M_{2}$ tidal constituents at ROBT, calculated for 19 successive individual years using T_TIDE's 't predic.m' from Pawlowicz et al. (2002) (a, b); and calculated continuously over 19 years using UTide's 'ut_reconstr.m' from Codiga (2011) (c, d)

due to the out-of-phase inter-annual variations in the diurnal versus semi-diurnal nodal factors (Fig. $5 \mathrm{c}$ ): resulting in use of near maximum values for the $\mathrm{O}_{1}$ and $\mathrm{K}_{1}$ nodal factors and a minimum value for the $\mathrm{M}_{2}$ nodal factor in this case.

For the nodal angles, the correction ranges (and minimum to maximum corrections) were $22.57^{\circ}$ (and $-11.12^{\circ}$ to $11.45^{\circ}$ ) for the $\mathrm{O}_{1}$ tide; $17.86^{\circ}$ (and $-8.97^{\circ}$ to $8.89^{\circ}$ ) for the $\mathrm{K}_{1}$ tide; and $4.48^{\circ}$ (and $-2.33^{\circ}$ to $2.15^{\circ}$ ) for the $\mathrm{M}_{2}$, respectively (Fig. $5 \mathrm{~d})$. Also, while 18.61 year cycle variation in the nodal factors was out-of-phase between the diurnal and semi-diurnal constituents (Fig. $5 \mathrm{c}$ ), the same period variations in the nodal angles were out of phase between the $\mathrm{O}_{1}$ tide versus both the $\mathrm{K}_{1}$ and $\mathrm{M}_{2}$ tides.

\section{Modifying the tidal prediction function in T_TIDE}

In short, we have found that T_TIDE is currently unable to produce accurate continuous multi-year predictions due to the 't predic.m' one-off calculation of NMC. To solve this issue, we made a small modification to 't $t$ predic.m' so that this function recalculates the NMC multiple times, with a default re-calculation interval of 1 day. This modified calculation procedure is illustrated in Fig. 3b, while Appendix 1 explains the modified 't predic.m' code, named 't predic_nmc.m'.

Using the modified 't predic_nmc.m' function, a 19 year continuous tidal height prediction was generated. As shown in Fig. $6 a$ and b, the predicted hourly tidal heights are able to accurately reproduce 18.61 year nodal cycle variations, due to the daily NMC updates. Further, the annual stepped variations produced by the original T_TIDE code are absent from these new and improved predictions, both in the diurnal components (compare Fig. $4 \mathrm{~b}$ and e with Fig. $6 \mathrm{~b}$ and e) and in the semi-diurnal components (compare Fig. $4 \mathrm{c}$ and $\mathrm{f}$ with Fig. 6c and f). 

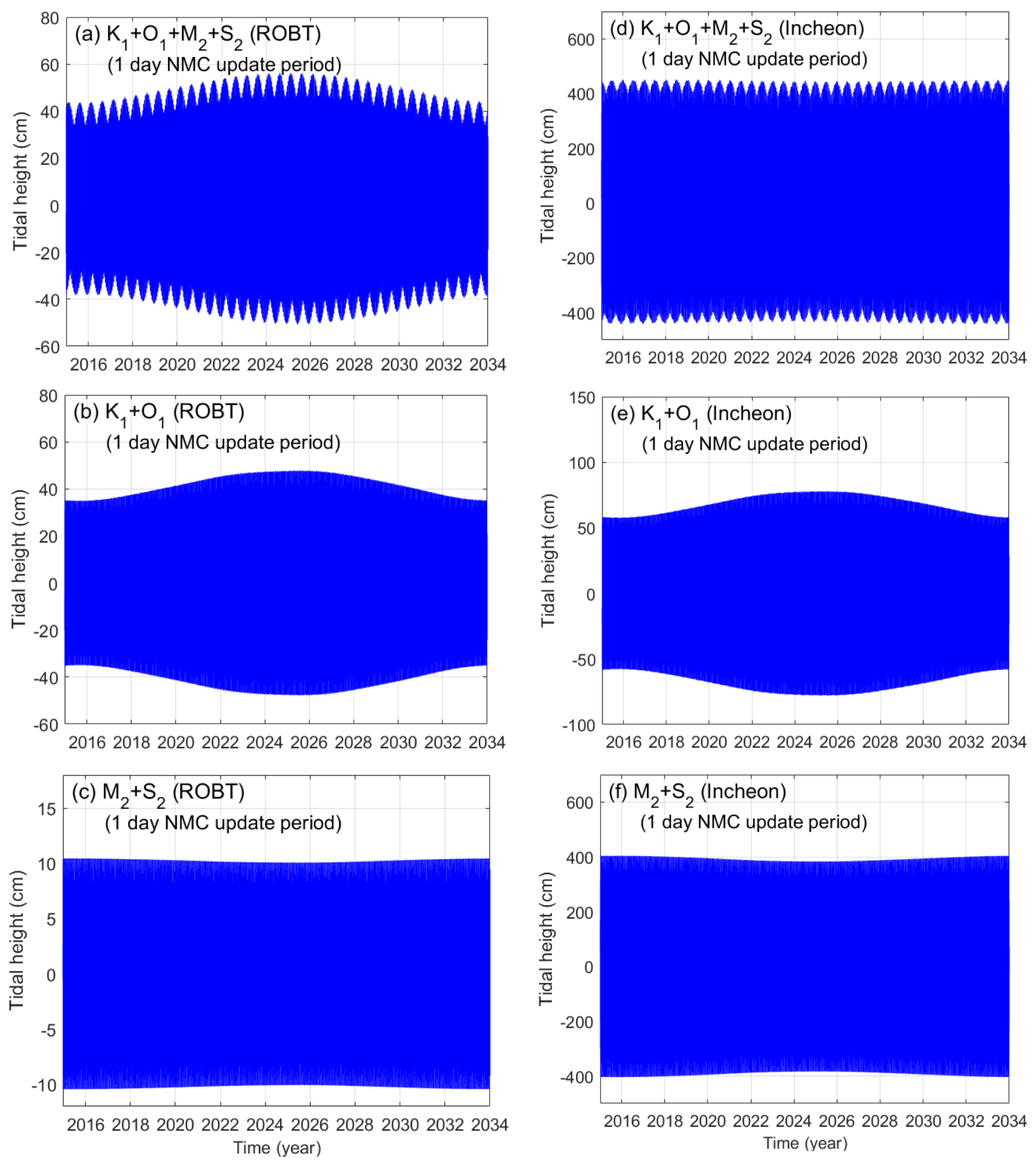

Fig. 6. Time series of continuous, hourly-interval tidal height predictions using the modified T_TIDE 't predic_nmc.m' function, with a one day nodal modulation correction (NMC) update period and using the four major tidal harmonic constants $(\mathrm{a}, \mathrm{d})$; the $\mathrm{K}_{1}$ and $\mathrm{O}_{1}$ harmonic constants only (b,e); and the $\mathrm{M}_{2}$ and $\mathrm{S}_{2}$ harmonic constants only (c,f), for the ROBT (a,b,c) and Incheon (d,e,f) tidal stations, respectively

\section{Summary}

A minimum 19 years of unbroken tidal harmonic predictions are required to determine LAT and HAT datums. In this study, we highlight and isolate a limitation in the widely used, classical tidal prediction package T_TIDE that hinders the generation of continuous multi-year tidal predictions. Via a simple modification to the nodal modulation correction update procedure in T TIDE's 't predic.m', we were able to solve the issue, enabling the generation of accurate, continuous 
19 year tidal predictions using this popular package.

Further, our results revealed that in tidally dominated coastal environments the NMC update period has a proportionally greater influence on the accuracy of longterm predictions for diurnal versus semi-semi-diurnal tides. This phenomenon is due to the greater level of nodal factor and nodal angle variation in diurnal versus semi-diurnal tidal constituents. Although proportionately smaller, the NMC effect on semi-diurnal tidal regimes should not be ignored in macro-tidal settings, as illustrated by the case of Incheon in Korea, since the NMC variations still produce significant absolute sea level variations in such places.

\section{Acknowledgments}

We thank the anonymous reviewers for very helpful comments, and gratefully thank Ms. Hyowon Kim for her kind assistance with Matlab script coding and the drafting of figures.

\section{References}

Byun D-S, Cho CW (2009) Exploring conventional tidal prediction schemes for improved coastal numerical forecast modeling. Ocean Model 28:193-202. doi:10.1016/j.ocemod.2009.02.001

Cartwright DE, Tayler RJ (1971) New computations of the tidegenerating potential. Geophys J Int 23:45-73
Cartwright DE, Edden AC (1973) Corrected tables of tidal harmonics. Geophys J Int 33:253-264

Codiga DL (2011) Unified tidal analysis and prediction using the UTide matlab functions. University of Rhode Island, Narragansett, $59 \mathrm{p}$

Courtier A (1938) Classification of tides in four types. In: Conférences sur les marées, Paris, pp 50-58

Foreman MGG (1977) Manual for tidal heights analysis and prediction. Institute of Ocean Sciences, Pacific Marine Science Report 77-10, $97 \mathrm{p}$

Foreman MGG, Cherniawsky JY, Ballantyne VA (2009) Versatile harmonic tidal analysis: Improvements and applications. J Atmos Ocean Tech 26:806-817 doi:10.1175/2008JTECHO615.1

Foreman MGG, Neufeld ET (1991) Harmonic tidal analyses of long time series. Int Hydrogr Rev LXVIII(I):85-109 (?????)

Leffler KE, Jay DA (2009) Enhancing tidal harmonic analysis: Robust (hybrid $\mathrm{L}^{1} / \mathrm{L}^{2}$ ) solutions. Cont Shelf Res 29:78-88. doi:10.1016/j.csr.2008.04.011

IHO (2018) Resolution on datums and benchmarks A2.5 3/1919. International Hydrographic Organization. https://www.iho. int/iho pubs/misc/M3-E-AUGUST18.pdf Accessed 5 Mar 2019

Pawlowicz R (2011) Rich Pawlowicz's matlab stuff. https://www. eoas.ubc.ca/ rich Accessed 5 Mar 2019

Pawlowicz R, Beardsley B, Lentz S (2002) Classical tidal harmonic analysis including error estimates in MATLAB using T_TIDE. Comput Geosci 28:929-937. doi:10.1016/S0098-3004(02)00013-4

Publisher's Note Springer Nature remains neutral with regard to jurisdictional claims in published maps and institutional affiliations. 


\section{Appendix}

Modified function ('t_predic_nmc.m') of 't_predic.m' in T_TIDE

$\%$ function yout $=\mathrm{t}$. predic(tim,varargin);

function yout=t_predic_nmc(tim,flag,nmcup,varargin);

\%\%\%\%\%\%\%\%\%\%\%\%\%\%\%\%\%\%\%\%\%\%\%\%\%\%\%\%\%\%\%\%

$\%$ This script describes a modified version of T_TIDE's t_predic.m

$\%$ incorporating flexible and frequent nodal modulation corrections (NMC),

$\%$ with a default update period of 1 day.

$\%$ This modification was developed to allow accurate multi-year

$\%$ (e.g., 19 year) tidal predictions using the T_TIDE package.

$\%$

$\%$ nmcup: update periods (unit: day) of $\mathrm{V}, \mathrm{u}$ and $\mathrm{f}$ (default 1 )

$\%$

$\%$ flag $=0$--> original $t$ predic (a central time for their $\mathrm{V}+\mathrm{u} \& \mathrm{f}$ calculations)

$\%=1$--> modified $\mathrm{t} \_$predic (a given time (nmcup) for their $\mathrm{V}+\mathrm{u} \& \mathrm{f}$ calculations)

$\%$ nmcup: update periods (unit: day) of $\mathrm{V}, \mathrm{u}$ and $\mathrm{f}$

$\%$

$\%$ For example (Incheon)

$\%$ Orignal case (flag =0): t $\_$predic_nmc (tim, 0, , tidestruc, 'latitude', 37.45);

$\%$ Modified case (flag $=1)$ : t_predic_nmc (tim, 1, 1, tidestruc, 'latitude', 37.45);

$\%$

$\%$ Do-Seong Byun 27/8/2019

$\%$

$\%$

$\%$ T_PREDIC Tidal prediction

$\%$ YOUT=T_PREDIC(TIM,NAMES,FREQ,TIDECON) makes a tidal prediction

$\%$ using the output of T_TIDE at the specified times TIM in decimal

$\%$ days (from DATENUM). Optional arguments can be specified using

$\%$ property/value pairs:

$\%$

$\%$ YOUT=T_PREDIC(...,TIDECON, property, value, ...)

$\%$

$\%$ Available properties are:

$\%$

$\%$ In the simplest case, the tidal analysis was done without nodal

$\%$ corrections, and thus neither will the prediction. If nodal

$\%$ corrections were used in the analysis, then it is likely we will

$\%$ want to use them in the prediction too and these are computed

$\%$ using the latitude, if given.

$\%$

$\%$ 'latitude' decimal degrees (+north) (default: none)

$\%$

$\%$ If the original analysis was $>18.6$ years satellites are

$\%$ not included and we force that here:

$\%$

$\%$ 'anallength' 'nodal' (default)

$\%$ 'full' For $>18.6$ years.

$\%$

$\%$ The tidal prediction may be restricted to only some of the

$\%$ available constituents:

$\%$

$\%$ 'synthesis' 0 - Use all selected constituents. (default)

$\%$ scalar $>0$ - Use only those constituents with a SNR
$\%$ greater than that given ( 1 or 2 are

$\%$ good choices).

$\%$

$\%$

$\%$ It is possible to call $t$ predic without using property names, in

$\%$ which case the assumed calling sequence is

$\%$

$\%$ YOUT=T_PREDIC (TIM, NAMES, FREQ, TIDECON, LATITUDE, SYNTHESIS);

$\%$

$\% \mathrm{~T}$ PREDIC can be called using the tidal structure available as an

$\%$ optional output from T_TIDE

$\%$

$\%$ YOUT $=$ T_PREDIC(TIM, TIDESTRUC, ...)

$\%$

$\%$ This is in fact the recommended calling procedure (and required

$\%$ when the analysis results are from series $>18.6$ years in length)

$\%$ R. Pawlowicz 11/8/99

$\%$ Version 1.0

$\% 8 / 2 / 03$ - Added block processing to generate prediction (to

$\%$ avoid memory overflows for long time series).

$\%$ 29/9/04 - small bug with undefined ltype fixed

if nargin $<3, \%$ Not enough

error('Not enough input arguments');

end;

longseries $=0$;

ltype $=$ 'nodal';

if isstruct(varargin $\{1\}$ ),

names $=$ varargin $\{1\}$.name;

freq $=$ varargin $\{1\}$.freq;

tidecon=varargin $\{1\}$.tidecon;

if isfield(varargin $\{1\}$,'Itype') \& strcmp(varargin $\{1\}$. ltyp(1:3),'ful'), longseries $=1$;

end;

$\operatorname{varargin}(1)=[]$;

else

if length(varargin) $<3$,

error('Not enough input arguments');

end;

names $=$ varargin $\{1\}$;

freq $=$ varargin $\{2\}$;

tidecon= $\operatorname{varargin}\{3\}$;

$\operatorname{varargin}(1: 3)=[]$;

end;

lat=[];

synth $=0$;

$\mathrm{k}=1$;

while length(varargin) $>0$,

if ischar(varargin $\{1\})$,

switch lower(varargin $\{1\}(1: 3)$ ), 

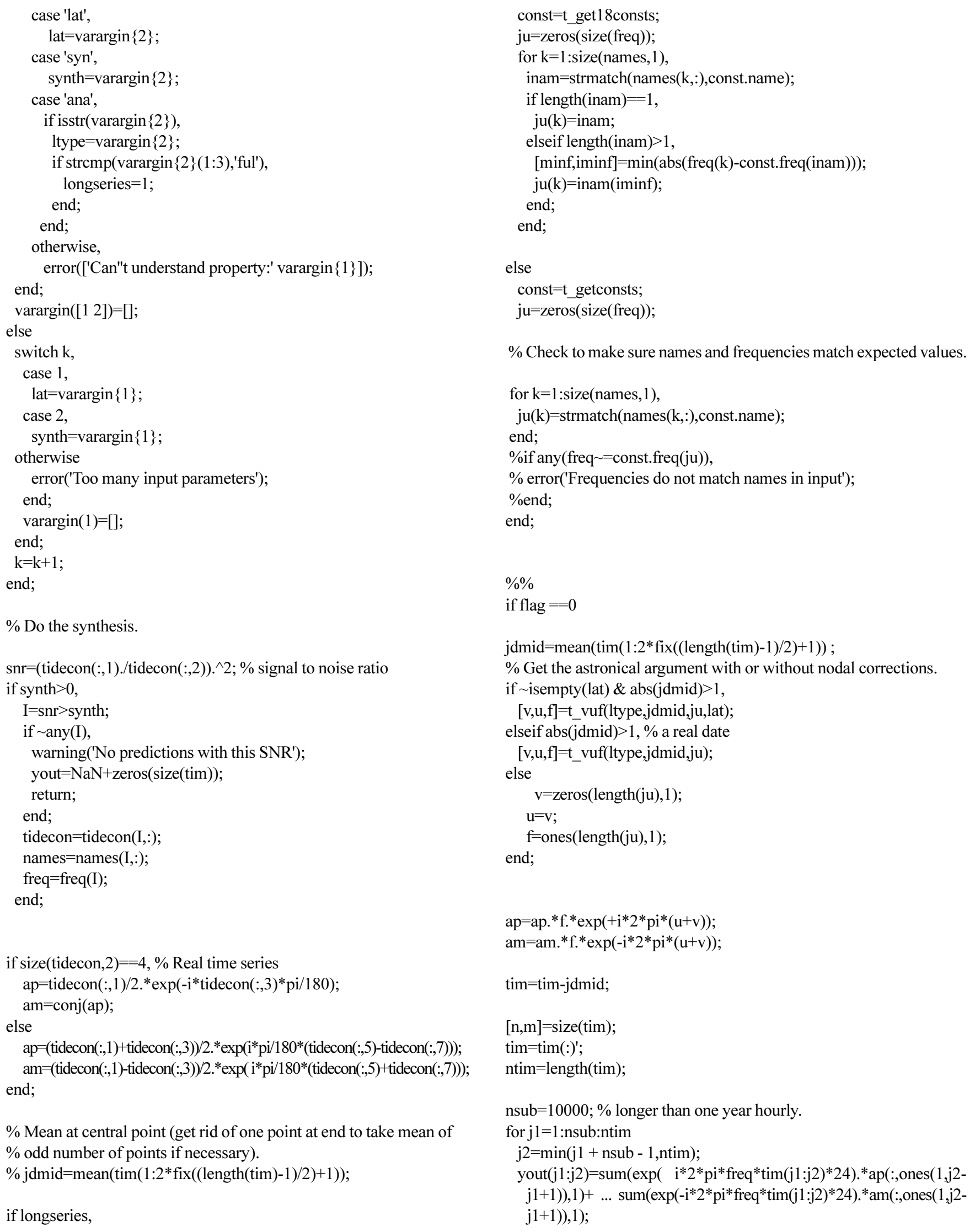
end;

elseif flag $==1$

\%\%\%\%\%\%\%\%\%\%\%\%\%\%\%\%\%\%\%\%\%\%\%\%\%\%\%\%\%\%\%\%

$\% \%$ This was included for the control of update periods

$\% \%$ of nodal modulation correction ( $\mathrm{u}$ and $\mathrm{f}$ ).

$\% \%$

$\% \%$ nmcup : update periods (unit: day) of $\mathrm{V}, \mathrm{u}$ and $\mathrm{f}$

$\% \%$ ndata : the number of data

$\% \%$ tsinx : the days at each starting index of update period

$\% \%$ tim_inx: time between each starting index

$\% \%$ March 9, 2019

\%\%\%\%\%\%\%\%\%\%\%\%\%\%\%\%\%\%\%\%\%\%\%\%\%\%\%\%\%\%\%\%

$\%$ nmcup $=1 ; \%$ every 1 day

if abs(tim(2)-tim(1) - 1/24/60)<0.0000001\% 1-min interval

ndata $=$ nmcup $* 24 * 60$;

elseif abs(tim(2)-tim(1) - 1/24/10) <0.0000001\% 6-min interval ndata $=$ nmcup $* 24 * 10$;

elseif abs(tim(2)-tim(1) - 1/24/6)<0.0000001 \%10-min interval ndata $=$ nmcup $* 24 * 6$;

elseif abs(tim(2)-tim(1) - 1/24) <0.0000001 \% 1-hr interval) ndata $=$ nmcup $* 24$

else

error('Check data time interval: $1 \mathrm{hr} / 10 \mathrm{~min} / 6 \mathrm{~min} / 1 \mathrm{~min}$ ') end

tsinx $=[1$ :ndata:length(tim) length(tim) +1$]$;

yout $=[]$;

$[\mathrm{n}, \mathrm{m}]=\operatorname{size}(\mathrm{tim})$;

for $\mathrm{ii}=1$ :length $(\operatorname{tsin} \mathrm{x})-1$

$\mathrm{idx}=\operatorname{tsin} x(\mathrm{ii}): \operatorname{tsin} x(\mathrm{ii}+1)-1$;

tim_inx $=$ tim(idx);

jdmid $=$ mean $($ tim_inx $(1: 2 *$ fix $(($ length $($ tim_inx $)-1) / 2)+1))$;

$\% \%$

$\%$ Get the astronical argument with or without nodal corrections. if isempty(lat) \& abs(jdmid) $>1$,

[v,u,f]=t_vuf(ltype,jdmid,ju,lat);

elseif abs(jdmid $>1, \%$ a real date

$[\mathrm{v}, \mathrm{u}, \mathrm{f}]=\mathrm{t} \_$vuf(ltype,jdmid,ju)

else

$\mathrm{v}=$ zeros(length(ju),1);

$\mathrm{u}=\mathrm{v}$;

$\mathrm{f}=$ ones(length(ju),1);

end;

ap1=ap. $*$ f. $* \exp \left(+\mathrm{i}^{*} 2 * \mathrm{pi}^{*}(\mathrm{u}+\mathrm{v})\right)$;

am1 $=$ am. $*$ f. $* \exp (-1 * 2 *$ pi*(u+v));

\%\%\%\%\%\%\%\%\%\%\%\%\%\%\%\%\%\%\%\%\%\%\%\%\%\%\%\%\%\%\%\%

$\% \%$ This was slightly modified. (March 9, 2019)

tim_inx=tim_inx-jdmid;

tim_inx=tim_inx(:)';

ntim=length(tim_inx);

yout_i $=[]$;

nsub $=10000 ; \%$ longer than one year hourly.

for $\mathrm{j} 1=1$ :nsub:ntim

$\mathrm{j} 2=\min (\mathrm{j} 1+$ nsub -1, ntim $)$;

yout_i $(\mathrm{j} 1: \mathrm{j} 2)=\operatorname{sum}\left(\exp \left(\mathrm{i}^{*} 2 * \mathrm{pi}^{*}\right.\right.$ freq*tim_inx $\left.(\mathrm{j} 1: \mathrm{j} 2) * 24\right) . *$ ap1(:,ones

$(1, j 2-j 1+1)), 1)+\ldots$ sum $\left(\exp \left(-i^{*} 2 * \mathrm{pi}^{*}\right.\right.$ freq $^{*}$ tim_inx $\left.(\mathrm{j} 1: \mathrm{j} 2) * 24\right) . * a m 1$ $(:$ ones $(1, \mathrm{j} 2-\mathrm{j} 1+1)), 1)$; end;

yout=[yout yout_i];

end;

\%\%\%\%\%\%\%\%\%\%\%\%\%\%\%\%\%\%\%\%\%\%\%\%\%\%\%\%\%\%\%\%

end

yout=reshape(yout,n,m); 